\title{
A NOTE ON THE THEORY OF THE FIRST VARIATION IN THE CALCULUS OF VARIATIONS.
}

\author{
By W. H. Young (Cambridge and Liverpool).
}

Adunanza del 26 dicembre 1909 .

I. In the modern treatment of the Calculus of Variations the Theory of Functions of a Real Variable inevitably plays a prominent part. Remarkable as has been, however, the progress of this latter theory in recent years, it may still be regarded as in its infancy, and still greater advances may be expected in the future. It seems therefore to be quite possible, in spite of the great attention which has been bestowed on the Calculus of Variations recently, that even the theory of the first variation of a simple integral has not yet assumed its final form.

In the present Note I propose to shew how a theorem in the Theory of Functions of a Real Variable, given by myself in these Rendiconti ${ }^{x}$ ), may be utilised to obtain a simple extension of the theory of the first variation, based on Du Bors Reymond's Lemma. The result proved is as follows:

If $f(x, y, z)$ possess with respect to each of the variables $y$ and $z$ partial differential coefficients which are continuous functions of $(x, y, z)$, and possess further a bounded differential coefficient with respect to $x$, and if $y=y(x)$ make the integral $\int_{x_{\mathrm{f}}}^{x_{2}} f\left(x, y, y^{\prime}\right) d x$ stationary, then the assumption that $y(x)$ possesses everywhere a bounded differential coefficient $y^{\prime}$ which is continuous EXCEPT AT MOST AT A COUNTABLE SET OF POINTS, insures the necessity of the equation

$$
f_{y^{\prime}}=c+\int_{x_{x}}^{x} f_{y} d x
$$

whence it follows, of course, that $f_{y^{\prime}}$ must be continuous everywhere, and that, except possibly at a countable set of points,

$$
\frac{d}{d x} f_{y^{\prime}}=f_{y} .
$$

Moreover, if we only consider solutions which make $f_{y^{\prime} y^{\prime}}$ everywhere different from zero, such a solution cannot have a differential coefficient everywhere which is disconti-

I) W. H. Young, A Theorem in the Theory of Functions of a Real Variable [Rendiconti del Circolo Matematico di Palermo, t. XXIV (2 ${ }^{\circ}$ sem. 1907), pp. I87-192. 
nuous at a countable set of points. In fact all solutions of the problem of making $\int_{x_{\mathrm{I}}}^{x_{2}} f\left(x, y, y^{\prime}\right) d x$ stationary, which possess a differential coefficient everywhere which is not a continuous function of $x$, must, if they satisfy $(A)$, also make $f_{y^{\prime} y^{\prime}}$ zero at the points of discontinuity, whether or not these be countable. Thus a discussion of such possible solutions involves precisely, when we come to the second variation, the case which is usually omitted from consideration, viz. that in which $f_{y^{\prime} y^{\prime}}$ is zero for the solution considered at one or more points ${ }^{2}$ ).

The notation adopted in the paper is essentially that used in BoLzA ${ }^{3}$ ).

2. We shall require the following lemma:

Lemma. - If $g(x, y, z)$ is a continuous function of the ensemble $(x, y, z)$, and if we substitute for $y$ any differentiable function of $x$ and for $z$ the differential coefficient of this function, supposed bounded in its ensemble, the function of $x$ so obtained assumes all values between its upper and lower bounds.

The proof of this lemma depends on the two facts:

I) that a differential coefficient assumes every value between its upper and lower bounds;

2) that the necessary and sufficient condition that a function of a single variable, which is the unique limit of a sequence of continuous functions, should assume all values between its upper and lower bounds, is that its value at any point is one of the limits for approach to the point on the right, and one of the limits for approach on the left [1.c. ' $)]$.

That the function $g\left[x, y(x), y^{\prime}(x)\right]$ in question is the limit of a continuous function appears if we write

$$
g\left[x, y(x), y^{\prime}(x)\right]=g\left[x, y(x), \mathrm{Lt}_{h=0} \frac{y(x+b)-y(x)}{b}\right] .
$$

But since $g(x, y, z)$ is a continuous function of $z$

$$
\operatorname{Lt}_{n=\infty} g\left(x, y, z_{n}\right)=g\left(x, y, \operatorname{Lt}_{n=\infty} z_{n}\right)=g(x, y, z),
$$

if $z_{n}$ describes any sequence of values having $z$ as unique limit.

Hence we may write

which proves the statement.

$$
g\left[x, y(x), y^{\prime}(x)\right]=\operatorname{Lt} g\left[x, y(x), \frac{y(x+h)-y(x)}{b}\right],
$$

We next shew that the value of $g\left[x, y(x), y^{\prime}(x)\right]$ at any point $x$ is a limit of its

2) The results of this paper may be compared with the important theorem recently proved by HAHN that every rectifiable curve which possesses a definite tangent at every point, necessarily satisfies equation $(B)$ if it furnishes a minimum for the integral under consideration. The work depends on the systematic use of generalised integration $\left\{\mathrm{H}_{\mathrm{AHN}}\right.$, Über die Herleitung der Differentialgleichungen der Variationsrechnung [Mathematische Annalen, Bd. LXIII (1907), pp. 253.272], p. 254\}.

3) O. BolzA, Vorlesungen über Variationsrechnung (Leipzig und Berlin, Teubner, I909). 
values in the neighbourbood both on the right and on the left. At a neighbouring point $x+b$ its value is $g\left[x+b, y(x+b), y^{\prime}(x+b)\right]$. Now by I) and 2 ) we can, for each fixed value of $x$, find such a sequence of values of $b$ with zero as limit that $y^{\prime}(x+b)$ has $y^{\prime}(x)$ for its unique limit. Also $x+b$ and $y(x+b)$ have respectively the unique limits $x$ and $y(x)$. Hence, since $g(x, y, z)$ is continuous with respect to the ensemble $(x, y, z)$ so that $g(x+b, y+k, z+l)$ has the unique limit $g(x, y, z)$ when $h, k$ and $l$ approach zero each in any manner, it follows that, choosing the particular sequence in question for the approach of $b$ to zero, $g\left[x+b, y(x+b), y^{\prime}(x+b)\right]$ has $g\left[x, y(x), y^{\prime}(x)\right]$ for unique limit.

Since $b$ may be either positive or negative this proves the statement made above. Thus $g\left[x, y(x), y^{\prime}(x)\right]$ has the two properties which, by 2) are sufficient to insure the truth of the lemma.

3. We have now to shew how the above lemma may be applied.

Proceeding in the usual way, we find that, in order that the integral $\int f\left(x, y, y^{\prime}\right) d x$ should have a stationary value it is necessary that

$$
\int_{x_{1}}^{x_{2}}\left(f_{y} n+f_{y^{\prime}} n^{\prime}\right) d x=0
$$

for all curves $n$ which possess a bounded differential coefficient $\eta^{\prime}$ and vanish in $x_{1}$ and $x_{2}$. Here we have used the fact that $f(x, y, z)$ possesses a first differential, which follows from the fact that $f_{x}$ exists and that $f_{y}$ and $f_{z}$ are continuous functions of $(x, y, z)$.

Writing

$$
u=n, \quad v=\int_{x_{1}}^{x} f_{y} d x,
$$

we remark that, since $\frac{d}{d y} f(x, y, z)$ is a continuous function of $(x, y, z), f_{y}$ is a continuous function of $x$, except for a countable set of values of $x$, and is bounded in its entirety.

Hence $\frac{d v}{d x}$ exists and is continuous except for a countable set of values of $x$, where it need not exist. Where it does exist, it is equal to $f_{y}$. It follows that $\int u \frac{d v}{d x} d x$ is the same as $\int u f_{y} d x$, if we interpret the symbol $\frac{d v}{d x}$ at a point where the differential coefficient does not exist to mean any one of the derivates of $v$. We may therefore write down the following formula,

$$
\int_{x_{\mathrm{I}}}^{x_{2}} u \frac{d v}{d x} d x=[u v]_{x_{\mathrm{I}}}^{x_{2}}-\int_{x_{\mathrm{I}}}^{x_{2}} v \frac{d u}{d x} d x
$$

and regard $\frac{d v}{d x}$ in this formula as standing for $f_{y}$. 
Equation (I) may therefore, applying this formula, be written in the form

$$
\int_{x_{1}}^{x_{2}} \eta^{\prime}\left(f_{y^{\prime}}-\int_{x_{1}}^{x} f_{y} d x\right) d x=0
$$

since $n$ vanishes at $x_{1}$ and at $x_{2}$.

4. We now require DU Bors ReYmond's well-known lemma in the following form:

If $N$ is a bounded function of $x$ such that

$$
\int_{x_{1}}^{x_{2}} n^{\prime} N d x=0
$$

for all functions $n$ which vanish at $x_{1}$ and $x_{2}$ and possess everywhere a differential coefficient, then the values of $N$ at all its points of continuity are the same.

If we further suppose that $N$ assumes in every interval all values between its upper and lower bounds in that interval, Hilbert's proof applies almost word for word as it stands, though he only contemplates the case when $N$ is continuous everywhere. If we do not make this assumption the change of wording is trivial. In the case to which we wish to apply the lemma, $N$ has the property in question.

5. Now write

$$
g\left(x, y, y^{\prime}\right)=f_{y^{\prime}}-\int_{x_{1}}^{x} f_{y} d x .
$$

Then evidently, since the integral on the right-hand side is necessarily a continuous function of its upper limit, and $f_{y^{\prime}}$ firstly is a continuous function of $x$ except at a countable set of points, secondly assumes all values in any interval between its upper and lower bounds in that interval, the same two properties are possessed by $g\left(x, y, y^{\prime}\right)$. But $g$ is the coefficient of $n^{\prime}$ in the equation (4), and may therefore be taken to be the $N$ of DU BoIs Reymond's Lemma. Hence $g$ has the same constant value except at a possible countable set of points. But $g$ assumes all values between its upper and lower bounds and therefore must either have a constant value or a set of values of potency $c$. Hence $g$ is constant, and we may write

$$
f_{y^{\prime}}-\int_{x_{1}}^{x} f_{y} d x=\text { const. }
$$

It hence follows that $f_{y^{\prime}}$ is continuous, and that, except possibly at the points at which $y^{\prime}$ is discontinuous, it possesses a continuous differential coefficient, viz. $f_{y}$. Thus, except at most at the points of the countable set in question we have the equation

$$
\frac{d}{d x} f_{y^{\prime}}=f_{y} \text {. }
$$

6. We have proved that $f_{y^{\prime}}$ is continuous even at the points where $y^{\prime}$ is discontinuous. In other words, taking a point at which $y^{\prime}$ is discontinuous, and where accordingly the limits of $y^{\prime}$ fill up an interval ${ }^{4}$ ), we get the same value for $f\left(y^{\prime}\right)$ - since $f(x, y, z)$

4) The limits at any point of a function which in every interval assumes all values between its upper and lower bounds necessarily include every value between the upper and lower limits at the point. 
is continuous with respect to the ensemble $(x, y, z)$ - whichever of these limits of $y^{\prime}$ we substitute for $z$ in $\frac{d f}{d z}$. In other words we have a pair of values of $x$ and $y$ and a whole interval of values of $z$ for which $\frac{d f}{d z}$ has the same value ${ }^{5}$ ). Therefore for this value of $x$ and this value of $y$ and any one of these values of $z, \frac{d^{2} f}{d z^{2}}$ exists and is zero ${ }^{6}$ ). Now since the value of the differential coefficient $y^{\prime}$ at the point is one of the limits of its values in the neighbourhood, it follows that we may take the $y^{\prime}$ at the point for the value of $z$ in question. Hence at any one of the points $x$ at which $y^{\prime}$ is discontinuous $f_{y^{\prime}, y^{\prime}}$ exists and is zero.

From this it follows that, if we exclude all solutions which make $f_{y^{\prime}, y^{\prime}}$ zero at one or more points, $y^{\prime}$ is necessarily continuous everywhere. On this hypothesis equation $(B)$ follows from equation $(A)$ without exception.

Thus we have shewn that the allowing of a countable set of possible discontinuities of $y^{\prime}$ will not enlarge the area of our possible solutions in the case under consideration. If on the other hand we do not exclude the possibility of $f_{y^{\prime}, y^{\prime}}$ vanishing at the points of discontinuity, the equation $(B)$ will then only hold with a possibly countable number of exceptional values of $x$.

7. It is scarcely necessary to point out that the considerations of the preceding article in no way depend on the assumption that the discontinuities of $y^{\prime}$ are countable. As long at least as $f_{y}$ is integrable whether in the RIEManN or in the generalised sense (summable), its integral is always a continuous function of its upper bound. As long as a function is bounded it may always be supposed without real restriction to possess a generalised integral, thus the equation $(A)$ has in this case always a meaning, and $f_{y^{\prime}}$ is necessarily a continuous function of $x$ if equation $(A)$ holds. If then we require, as we usually do, that $f_{y^{\prime}, y^{\prime}}$ should not be zero anywhere for the solution in question, we are sure a priori that no solution whose differential coefficient has discontinuities, whether finite in number or countably or more than countably infinite, can possibly exist, for which equation $(A)$, still less its derived equation, is true.

5) For if $g(x, y, z)$ is a continuous function of $(x, y, z)$ which becomes a function $G(x)$ of $x$, continuous at $x=x_{0}$, when we make $y=y(x)$ and $z=z(x)$, functions of $x$ of which the first is continuous and the second discontinuous at $x=x_{0}$, and $k$ denote any one of the limits of $z(x)$ at $x=x_{0}$, we can find a sequence of values of $x$ with $x_{0}$ as limit, such that $z(x)$ has the unique value $k$. Therefore the point $[x, y(x), z(x)]$ will describe a sequence having the point $\left(x_{0}, y_{0}, k\right)$ as limiting point. Since $g(x, y, z)$ is continuous, $g[x, y(x), z(x)]$ will then have the unique limit $g\left(x_{0}, y_{0}, k\right)$. But since $G(x)$ is continuous at $x=x_{0}, g[x, y(x), z(x)]$, that is $G(x)$, will have the unique limit $G\left(x_{0}\right)$, that is $g\left(x_{0}, y_{0}, z_{0}\right)$. Thus

$$
g\left(x_{0}, y_{0}, k\right)=g\left(x_{0}, y_{0}, z_{0}\right) \text {. }
$$

6) If $z^{\prime}$ is an end-point of the interval of limits in question, $\frac{d f}{d z}$ will be a differential coefficient on one side which has the constant value in question so that if $\frac{d^{2} f}{d q^{2}}$ exists there, it is zero. 
On the other hand we see, if we are to completely discuss the possibility of solutions possessing a differential coefficient everywhere, which is not necessarily continuous, we cannot in considering the second variation, confine our attention, as is commonly done in the theory, to the case where $f_{y^{\prime}, y^{\prime}}$ never vanishes.

8. We may apply the above theory like the ordinary theory. Consider the cases where $f(x, y, z)$ does not contain $y$, or does not contain $x$, or is a linear function of $z$. It is obvious that in the first case equation $(A)$ gives us at once the usual equation

$$
f_{y^{\prime}}=\text { const., }
$$

so that in this case $(B)$ also holds for all values of $x$.

Next considering the third case, write

$$
f(x, y, z)=L(x, y)+z M(x, y),
$$

where, in accordance with the assumptions with respect to $f(x, y, z)$ already made, $L$ and $M$ have necessarily differential coefficients with respect to $x$ and $y$, of which the latter are continuous with respect to $(x, y)$. Equation $(B)$ now takes the form

$$
M_{x}+y^{\prime} M_{y}=L_{y}+y^{\prime} M_{y}
$$

whence, except possibly at a countable set of points,

$$
M_{x}=L_{y}
$$

If therefore we make the additional assumption that $M_{x}$ is continuous with respect to $(x, y)$, this equation will still hold at the doubtful points, and therefore the equation $(B)$ will be true without exception, as in the ordinary theory.

In the discussion of the second case, that in which $f$ does not contain $x$ explicitly, it is convenient to at once make the additional assumption that $f_{y^{\prime}, y^{\prime}}$ exists and to consider only such possible solutions as make this different from zero for every value of $x$. It is then at once obvious, as HILBERT has pointed out, that when $y^{\prime}$ is continuous, $y^{\prime \prime}$ also exists. On our assumptions $y^{\prime}$ is not continuous everywhere but it follows in precisely the same way that where it is continuous $y^{\prime \prime}$ exists.

Hence at all but a countable set of points we have

$$
\frac{d}{d x}\left(f-y^{\prime} f_{y^{\prime}}\right)=y^{\prime} f_{y}+y^{\prime \prime} f_{y^{\prime}}-y^{\prime} \frac{d}{d x}\left(f_{y^{\prime}}\right)-y^{\prime \prime} f_{y^{\prime}}=0 .
$$

But since a differential coefficient assumes all values between its upper and lower bounds it can only have a single value or a set of values of potency $c$. Hence the preceding equation holds for all values of $x$ without exception, from which moreover it follows that equation (2) holds always, and the theory differs in no way from the ordinary theory ${ }^{7}$ ).

Genève, La Nonette de la Forêt, December $18^{\text {th }}$, I 909.

\section{W. H. Youn G.}

7) I take this opportunity of referring the readers of the Rendiconti who may have read the remarks of Schoenflies on a former paper of mine in the present Rendiconti, Tomo XXI (1906), pp. 125-127 to my reply in the Messenger of Mathematics, New Series, $\mathrm{n}^{\circ} 46 \mathrm{I}$, September rgog. Readers who, like Schoenflies, may have found the argument difficult to follow, will find it there expanded, though in no other way altered. 